\title{
Microbubble and ultrasound radioenhancement of bladder cancer
}

\author{
WT Tran ${ }^{1,2,3,4}$, S Iradji ${ }^{2,3}$, E Sofroni ${ }^{2,3}$, A Giles $^{2,3}$, D Eddy ${ }^{4}$ and GJ Czarnota ${ }^{*, 1,2,3,5}$ \\ 'Department of Radiation Oncology, University of Toronto, Toronto, Ontario, Canada; ${ }^{2}$ Department of Imaging Research, Sunnybrook Health Sciences \\ Centre, 2075 Bayview Avenue, Toronto, Ontario, Canada; ${ }^{3}$ Department of Radiation Oncology, Sunnybrook Health Sciences Centre, 2075 Bayview \\ Avenue, Toronto, Ontario, Canada; ${ }^{4}$ Department of Radiotherapy and Oncology, Sheffield Hallam University, Howard Street, Sheffield, South Yorkshire SI \\ IWB, UK; ${ }^{5}$ Department of Medical Biophysics, University of Toronto, Toronto, Ontario, Canada
}

\begin{abstract}
BACKGROUND: Tumour vasculature is an important component of tumour growth and survival. Recent evidence indicates tumour vasculature also has an important role in tumour radiation response. In this study, we investigated ultrasound and microbubbles to enhance the effects of radiation.

METHODS: Human bladder cancer HT-1376 xenografts in severe combined immuno-deficient mice were used. Treatments consisted of no, low and high concentrations of microbubbles and radiation doses of 0, 2 and $8 \mathrm{~Gy}$ in short-term and longitudinal studies. Acute response was assessed $24 \mathrm{~h}$ after treatment and longitudinal studies monitored tumour response weekly up to 28 days using power Doppler ultrasound imaging for a total of 9 conditions ( $n=90$ animals).

RESULTS: Quantitative analysis of ultrasound data revealed reduced blood flow with ultrasound-microbubble treatments alone and further when combined with radiation. Tumours treated with microbubbles and radiation revealed enhanced cell death, vascular normalisation and areas of fibrosis. Longitudinal data demonstrated a reduced normalised vascular index and increased tumour cell death in both low and high microbubble concentrations with radiation.

CONCLUSION: Our study demonstrated that ultrasound-mediated microbubble exposure can enhance radiation effects in tumours, and can lead to enhanced tumour cell death.

British Journal of Cancer (2012) 1 07, 469-476. doi:I0.1038/bjc.2012.279 www.bjcancer.com

Published online 12 July 2012

(c) 2012 Cancer Research UK
\end{abstract}

Keywords: cell death; apoptosis; ultrasound; anti-vascular therapy; bladder cancer; radiosensitizers

The meshwork of blood vessels within the tumour microenvironment is constituted in part by endothelial cells that proliferate as part of the recruitment of new vessels to the tumour. These cells are critical in response to both intracellular and extracellular signals that initiate neovascularization. Anti-vascular agents that target endothelial cells are primarily focused on inhibition of angio-regulators such as vascular endothelial growth factor (VEGF), angiogenin and thrombin (Hayes et al, (1999)). Antivascular pharmacological agents have been successful in preclinical trials although there are several limitations to their application as a monotherapeutic approach (O'Reilly, 2006). These limitations include the requirement to administer a high dose within a prolonged treatment course in order to achieve optimal therapeutic efficacy (O'Reilly, 2006) and obstacles in targeting tumour vasculature only to spare normal vasculature in routine physiological processes (Thorpe, 2004). Phase I studies have reported toxicities associated with the administration of these pharmacological agents such as anorexia, constipation, dyspnoea, fatigue, headache, pain and hypokalemia in addition to rebound tumour growth after the cessation of therapy (Gadgeel et al, 2002; Radema et al, 2002; Jameson et al, 2003; Rustin et al, 2003; Thorpe, 2004). In the present study, we use ultrasound-mediated microbubbles as a novel treatment to perturb endothelial cells within the vascular framework in vivo.

*Correspondence: Dr G] Czarnota; E-mail: gregory.czarnota@sunnybrook.ca Received 23 January 20 12; revised 29 May 20 I2; accepted 30 May 20 I2; published online 12 July 2012
There is a growing body of work suggesting that there can be synergistic anti-tumour effects when anti-vascular agents are combined with radiation. Recent reports propose radiation response as a function of the tumour vasculature (Raben and Ryan, 2005; Nieder et al, 2006; Dings et al, 2007). This is in addition to the classically accepted model that radiosensitivity and tumour control is a result of direct and indirect effects of ionising radiation on tumour stem cells and undifferentiated progenitor clonogens. Vascular damage has been long reported as an inherent effect when tumours are exposed to ionising radiation (Thomlinson, 1973). Therefore, there is an opportunity to further investigate the impact of antivascular agents and radiation to the tumour microenvironment.

Microbubble microspheres are coated with a lipid, protein or biopolymer, and are heavily used in medical applications of ultrasound. These microspheres encapsulate a specific gas that give them characteristic echogenicity and are currently used as contrast agents in ultrasound diagnostic imaging. Evidence that endothelial cells can be damaged during ultrasound-mediated microbubble exposure have been reported and demonstrated that endothelial cells were susceptible bystanders to injury from cavitation (Kobayashi et al, 2002). Recent investigations suggest potential in exploiting ultrasound-driven microbubbles for drug delivery in cancer therapeutics because of their affinity to permeate the endothelium of tumour microvessels (Klibanov, 2006). Advances in medical oncology are hence focusing on using microbubbles to deliver agents that would sensitise tumours to chemotherapeutic drugs by interfering with the microvasculature. Similar principles of angio-targeting are being applied to research in radiation oncology and here, we investigate microbubbles in this capacity. 
A major effect caused by ultrasound interactions with microbubbles can be cavitation. Cavitation is described as rapid oscillating movements and morphological transformations that can result in outer-shell disruption of microbubbles and gas release. In cardiovascular medicine ultrasound-driven microbubbles can disrupt red blood clots, making it an effective therapy for the treatment of intravascular thrombin (Xie et al, 2005). Yu et al, 2004 reported that ultrasound-driven microbubbles could irreparably damage kidneys locally. They concluded that this modality could potentiate cell rupture from microsphere insonification in vivo (Xie et al, 2005). Previous studies have reported ultrasound-mediated microbubbles exposure to be efficacious in perturbing endothelial cells (Kobayashi et al, 2002). Endothelial cells have an important role in the vascularisation process. De novo vascularisation is reliant on the recruitment of endothelial progenitor cells that originate from the bone marrow and occur in wound healing, arthritis, inflammation and tumourigenesis (Nieder et al, 2006). Neoplastic conversion is followed by several biochemical signals that induce vascular bridging into the tumour matrix and is required for tumours to grow beyond $2 \mathrm{~mm}$ (Nishida et al, 2006; Xuan et al, 2007). Vasculature in tumours is atypical from normal vessels resulting in haemostatic imbalance. These vessels are immature, leaky and are inefficient at supplying oxygen and nutrients (Jain, 2005; Fukumura and Jain, 2007) beyond $90-140 \mu \mathrm{m}$. Tumour cells beyond this proximal distance adapt by modifying their activities and give rise to an acidic and hypoxic tumour environment (Cooney et al, 2006).

Power Doppler ultrasound is an important imaging tool used to investigate and map vascular structures by detecting flow of red blood cells. Power Doppler sonograms can be created by measuring echoes from moving scatterers (red blood cells that create phase shifts that are used to calculate the vascular landscape). Its advantages over other ultrasonographic imaging approaches rely on its ability to overcome common obstacles such as angle dependence, aliasing and filtering background noise (Hamper et al, 1997).

This study used high-resolution power Doppler ultrasound to monitor overall tumour response as a result of induced local damage to the tumour microvessels. We employed ultrasoundmediated microbubbles as a vascular targeting agent in combination with radiation. This study utilised a bladder cancer model as radiation can be used as a clinically definitive bladder sparing therapy and as an adjuvant modality to chemotherapy and surgery. The results of this study demonstrate that concomitant treatment with radiation and microbubbles has the ability to potentiate vascular damage by close to four-fold in certain treatment conditions in vivo. Tumours exhibited large areas of cell death caused by ultrasound-mediated microbubble exposure. The study results suggest that ultrasound-mediated microbubble treatment combined with radiation may be useful in destabilising tumour vasculature and enhancing radiation response in bladder cancer xenografts in vivo.

\section{MATERIALS AND METHODS}

\section{Cell culture}

Cell lines were obtained from the American Type Culture Collection (ATCC, Manassas VA, USA). Human bladder carcinoma HT-1376 cell lines were cultured in Eagle's minimum essential medium (ATCC) supplemented with $10 \%$ foetal bovine serum (Sigma-Aldrich, St Louis, MO, USA), 1\% penicillin/streptomycin (Sigma-Aldrich) and exposed to $5 \% \mathrm{CO}_{2}$ hepa-filtered air at $37^{\circ} \mathrm{C}$. Cells were cultured to $80 \%$ confluence and collected using $0.25 \%$ trypsin, $0.02 \%$ EDTA solution at room temperature. Cell pellets were isolated and re-suspended in $100 \mu \mathrm{l}$-PBS $(\mathrm{Mg}-, \mathrm{Ca}-)$ per $1.0 \times 10^{6}$ cells in preparation for inducing tumours in mice.

\section{Animals}

Animal research was conducted in accordance with the guidelines by the Canadian Council on Animal Care. CB-17 white-haired severe combined immuno-deficient (SCID) male mice were obtained from Charles River Inc. (Wilmington, MA, USA). A total cell volume of $1.0 \times 10^{6}$ cells suspended in $100 \mu$ of D-PBS $(\mathrm{Mg}-$, $\mathrm{Ca}-$ ) was injected subcutaneously to the lower right hind leg of the mouse and tumours were allowed to develop over a period of 2-3 weeks in order to reach a diameter of 5-7 $\mathrm{mm}$ for experiments.

\section{In vivo image acquisition and analysis}

Blood flow was detected using a VEVO770 ultrasound unit (VisualSonics, Toronto, Ontario, Canada) in power (amplitude) Doppler mode with a $25-\mathrm{MHz}$ transducer (Visualsonics RMV$710 \mathrm{~B}$, centre frequency $=20 \mathrm{MHz}$, lateral resolution $=149 \mu \mathrm{m}$, axial resolution $=54 \mu \mathrm{m}$, (manufacturer specifications)). The real-time microvisualization transducer was employed to acquire 3D volumetric scans (scan speed $2.0 \mathrm{~mm} \mathrm{~s}^{-1}$, wall filter $2.5 \mathrm{~mm} \mathrm{~s}^{-1}$ and step size of $0.2 \mathrm{~mm}, 90 \%$ bandwidth, $12.8 \mathrm{~mm}$ focal depth) where the centre of the tumour was placed at the acoustic focus. Mice were anaesthetized using a combination of $2 \%$ oxygen ventilated isoflurane for induction and then with i.p. injection of ketamine $100 \mathrm{mg} \mathrm{kg}^{-1}$, xylazine $5 \mathrm{mg} \mathrm{kg}^{-1}$ and acepromazine $1 \mathrm{mg} \mathrm{kg}^{-1}$ in $0.9 \%$ sodium chloride saline titrated at $0.02 \mathrm{ml}$ intervals to a maximum dose of $0.1 \mathrm{ml}$. All mice were imaged before treatment administration in order to assess baseline microvessel blood flow for both acute and longitudinal studies. For 'acute' studies, the mice were scanned $24 \mathrm{~h}$ after treatment. For 'longitudinal' studies, the mice were scanned at $24 \mathrm{~h}$ and $7,14,21$ and 28 days as established by animal health endpoints criteria. The number of images acquired per scan ranged from 60-100 frames depending on tumour size. Power Doppler images were converted into device-independent binary data for quantitative analysis using custom designed software with MATLAB (Mathworks Inc., Natick, MA, USA). Microvessel flow signals were determined by selecting a region of interest (ROI) encompassing the whole tumour in each frame. Each frame was constructed by quantised colour and non colour voxels. The vascular index (VI) was calculated by dividing the total number of colour voxels (representing the power Doppler signal from red-blood cell backscatters) by the non colour voxels (representing non-vascular regions). The normalised VI (NVI) was calculated by comparing the VI at each time point against the baseline VI.

In order to minimise experimental variability, each experimental group of animals (treatment type) had imaging carried out within the same session. To minimise variability during acquisition settings, the tumour-bearing hind leg was immersed in a constant temperature water bath to couple the transducer to the tumour. The water used was degassed in advance through vacuum pressure before using for experiments to minimise air bubbles, which interfere with imaging. Titrated anaesthetic was administered in order to ensure that the animal's blood pressure was maintained and to also reduce any respiratory and cardiac motion artifacts on the Doppler signal. The mouse's body temperature was maintained to reduce vasoconstriction by keeping it on a heating pad with its core temperature monitored rectally. Animals used for experiments were similar in age, size and weight. Any data frames that had motion artifacts were removed from analysis.

\section{In vivo studies}

Microbubbles and ultrasound activation Definity Perflutren lipid microspheres (Lantheus Medical Imaging, North Billerica, MA, USA) were shaken using a Lantheus device for $45 \mathrm{~s}$ at 3000 r.p.m. Low $(1 \% \mathrm{v} / \mathrm{v})$ and high $(3 \% \mathrm{v} / \mathrm{v})$ microbubble concentrations were calculated according to total mouse blood volume estimated by 
animal weight. The microbubbles were diluted in sterile normal saline and injected via the tail vein. A secondary injection $(0.1 \mathrm{cc})$ of normal saline was used to flush the tail vein before treatment. Mice were mounted onto a custom stage and partially immersed into a $37^{\circ} \mathrm{C}$ water bath for ultrasound exposures. The ultrasound therapy system involved a micro-positioning system, waveform generator (AWG520, Tektronix, Beaverton, OR, USA), power amplifier with pulser/receiver (RPR4000, Ritec) and a digital acquisition system (Acquiris CC103, Agilent Technologies, Monroe, NY, USA). Animals were exposed within the half maximum peak of the acoustic signal $(-6 \mathrm{~dB}$ beam width of $31 \mathrm{~mm}$ and depth of field $>2 \mathrm{~cm}$ ) 16 -cycles tone burst at $500 \mathrm{kHz}$ centre frequency using a $2.85-\mathrm{cm}$ unfocused planar ultrasound transducer (ValPey Fisher Inc., Hopkinton, MA, USA) and at $3 \mathrm{kHz}$ pulse repetition frequency for $50 \mathrm{~ms}$ at a time with a peak negative pressure set to $570 \mathrm{kPa}$, corresponding to a mechanical index of 0.8 (Karshafian et al, 2009). An intermittent 1950-ms period between sonification was employed to minimise biological heating in the tissue during ultrasound exposures. The total insonification time was $750 \mathrm{~ms}$ over $5 \mathrm{~min}$.

Irradiation The tumours were $\mathrm{X}$-irradiated $5 \mathrm{~min}$ after ultrasound treatment using an irradiation cabinet device (Faxitron, Wheeling, IL, USA). Doses of 0,2 and 8 Gy were administered at a dose rate of $200 \mathrm{cGy} \mathrm{min}^{-1}, 160 \mathrm{kVp}$ energy and a source-skin distance of $30 \mathrm{~cm}$ as per the specifications of the device. Corporal lead sheet shielding was used with a circular aperture to expose only the tumour.

\section{Histopathology}

Tumours were fixed in $10 \%$ acetate buffered formalin (Fisher Scientific Canada, Ottawa, Ontario, Canada) following excision at $24 \mathrm{~h}$ (for acute studies) and 21-28 days (for long-term studies) after treatment. Tumours were fixed at room temperature for $4 \mathrm{~h}$ and then transferred to $4{ }^{\circ} \mathrm{C}$ for $24 \mathrm{~h}$ before processing using a Leica ASP300 smart tissue processor (Leica Microsystems, Richmond Hill, Ontario, Canada). Tissues were embedded in paraffin (Leica EG 1160, Leica Microsystems) and prepared as $5 \mu \mathrm{m}$ sections onto slides with standard hematoxylin and eosin (H\&E) staining techniques used for both acute and longitudinal studies. Staining using TdT-mediated dUTP-biotin nickend-labelling (TUNEL) was used in acute studies to visualise apoptotic regions. Cluster of differentiation-31 (CD31) staining was implemented to count and quantify endothelial cells within the tumour relative to the presence of intact and disrupted vessels.

Specifically, for microscopy of specimens on slides, a Leica DC100 microscope was used with a $\times 20$ objective coupled to a 1-MPixel Leica DC100 video camera wired to a $2-\mathrm{GHz}$ PC running Leica IM1000 software (Leica Microsystems GmbH, Wetzlar, Germany). A normalised CD31 VI was calculated using 10 randomly selected ROIs per tumour slice from five slices per animal tumour. The VI was calculated as the ratio of the sum of intact luminal vessel number/area measured to the total vessel number/area measured (including intact luminal vessels and vessels which had been ruptured or collapsed by microbubble exposure). Vessels stained with CD31, in addition to areas of cell death, and all other microscopy measures were quantified in histology and immunohistochemistry tumour sections assisted by the use of Image-J (NIH, Bethesda, MD, USA).

\section{Statistical analysis}

A detailed analysis was conducted using GRAPHPAD INSTAT (GraphPad Software Inc., La Jolla, CA, USA) and a statistician was consulted to review the most appropriate statistical method. Oneway analysis of variance statistical analysis with Dunnett's test or Tukey's multiple comparison's test was performed.

\section{RESULTS}

\section{Microbubbles alone effectively target vasculature in HT-1376 bladder cancer xenografts in vivo}

In order to quantify vasculature damage as a result of ultrasound-microbubble treatments alone, HT-1376 bladder cancer xenografts were treated with low and high concentration of microbubbles and exposed to ultrasound positioned over the tumour. The acute studies revealed that HT-1376 bladder carcinoma microvessel were responsive to treatments and demonstrated a reduction in the mean power Doppler index from the initial measured baseline. Low-concentration microbubble-ultrasound treatment after $24 \mathrm{~h}$ revealed a NVI was $0.91 \pm 0.01$ (s.e.m.), representing a reduction in vasculature by $\sim 8.6 \%$ (Figure $1 \mathrm{~B}$ ). The higher microbubbleultrasound treatment resulted in a NVI of $0.85 \pm 0.01$ or a reduction in vasculature by $15 \%$ (Figure 1C). The longterm studies revealed similar results. Tumours that were treated with 1 and $3 \%$ microbubble concentrations when driven by ultrasound resulted in a mean power Doppler measurement of $0.71 \pm 0.01$ and $0.70 \pm 0.01$, respectively, after 21 days (Figures $2 \mathrm{~B}$ and $\mathrm{C})$.
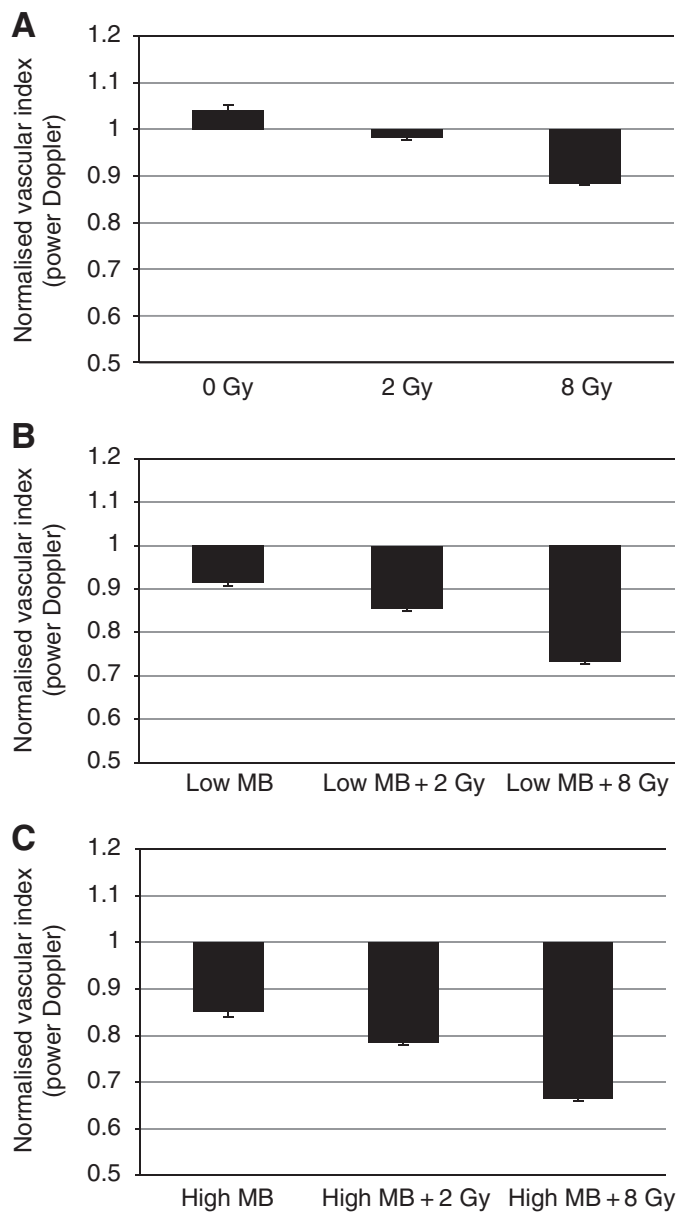

Figure I Twenty four hours response monitoring of tumour vasculature using power Doppler ultrasound. (A) Tumours exposed to increasing doses of radiation demonstrated reduction in the vascularity $(P<0.01$, $n=5$ ). (B, C) Combination therapies with low and high concentration of ultrasound-activated microbubbles and radiation. Normalised vascular index using power Doppler ultrasound was used to measure vessel abundance $(P<0.01, n=5)$. Error bars $=$ s.e.m. 
The combination of ultrasound-driven microbubbles and radiation synergistically decreases vasculature in HT-1376 bladder cancer tumours in vivo

In order to explore how radiation alone affected the vasculature, HT-1376 bladder cancer tumours were exposed to 2 and $8 \mathrm{~Gy}$ of ionising radiation as experimental controls. The findings revealed that the tumour vasculature was diminished in flow within the first $24 \mathrm{~h}$ of treatment, primarily at the $8 \mathrm{~Gy}$ dose $(\mathrm{NVI}=0.88 \pm 0.00)$ and negligibly at the $2 \mathrm{~Gy}$ dose $(\mathrm{NVI}=0.98 \pm 0.00)$ (Figure $1 \mathrm{~A})$. In our long-term studies, a negligible detected increase in flow at 2 Gy was observed (NVI of $1.04 \pm 0.00$ ) and the 8 Gy dose reached a NVI of $0.60 \pm 0.01$ (Figure 2A).

Results indicated that combination treatments with microbubbles and radiation lead to synergistic anti-vascular effects as observed by power Doppler and immunohistochemistry. Short-term studies $(24 \mathrm{~h})$ demonstrated that at low microbubble concentration, there was a decrease in detectable flow with $2 \mathrm{~Gy}$ administration $(\mathrm{NVI}=0.86, \pm 0.00)$ and with 8 Gy $(\mathrm{NVI}=0.73 \pm 0.00)$ compared with the baseline (Figure 1B). The higher microbubble concentration revealed a further decrease in detectable blood flow when combined with $2 \mathrm{~Gy}$
$(\mathrm{NVI}=0.79 \pm 0.01)$ and 8 Gy $(\mathrm{NVI}=0.66 \pm 0.00)$, which was greater than with microbubbles alone (Figure 1C).

Long-term studies showed similar reduction in vascularity when measured at 21 days. When $2 \mathrm{~Gy}$ was combined with the low microbubble concentration treatment, a NVI of $0.50 \pm 0.01$ was observed and at $8 \mathrm{~Gy}$ with low-concentration microbubbles, a NVI of $0.43 \pm 0.00$ was observed (Figure 2B). When $2 \mathrm{~Gy}$ radiation was combined with high-concentration microbubbles a NVI of $0.44 \pm 0.01$ was observed. When $8 \mathrm{~Gy}$ radiation was administered with the high concentration of microbubbles in the presence of ultrasound a NVI of $0.34 \pm 0.01$ was observed (Figure 2C). These changes show significant synergistic treatment effects when compared with treatment of ultrasound-driven microbubbles alone.

\section{The combination of ultrasound-driven microbubbles} and radiation significantly enhances tumour cell death in the tumour's centre compartment caused by endothelial cell disruption

We tested to determine the efficacy of microbubbles in combination with radiation to induce vascular atrophy and to enhance

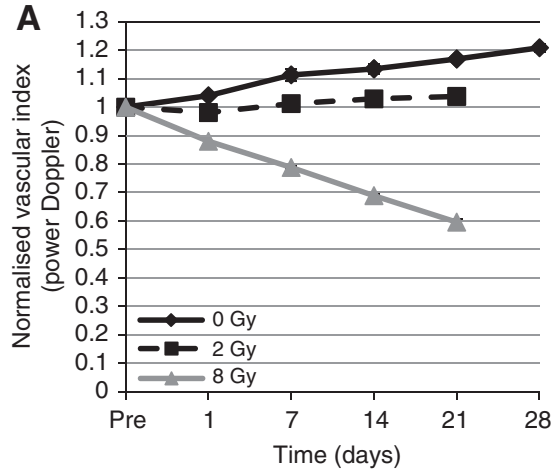

D
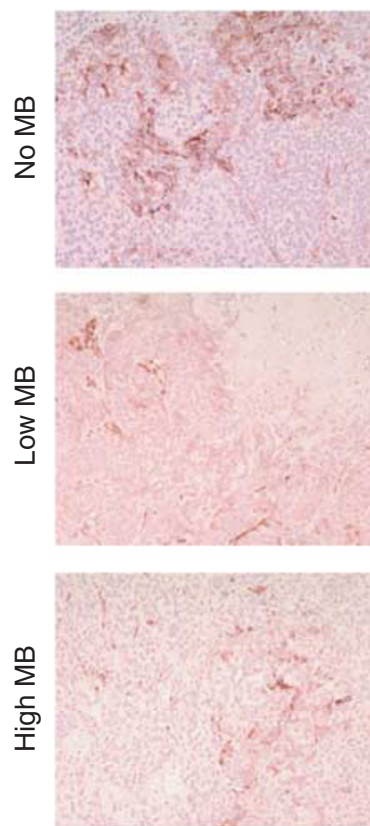

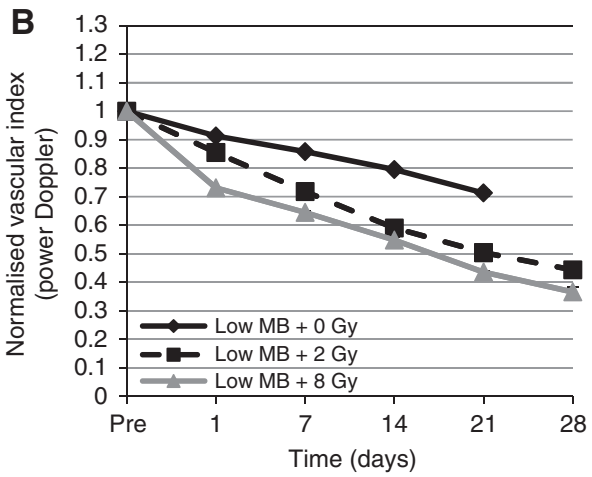

2 Gy
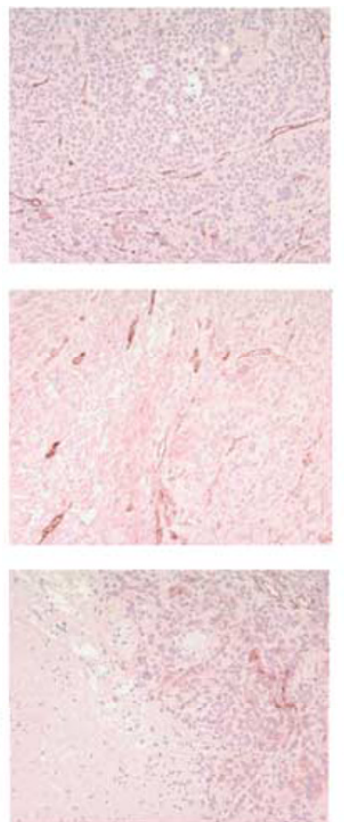

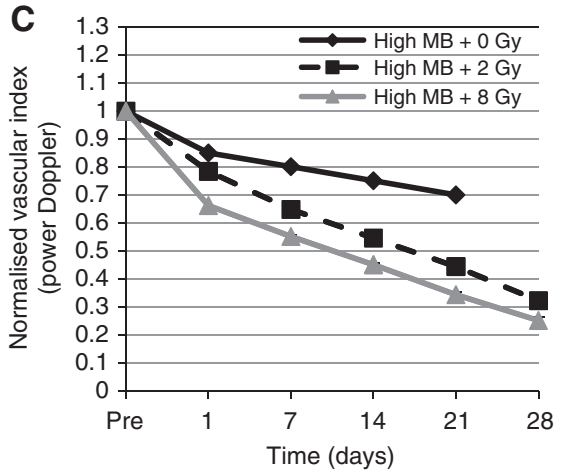

8 Gy
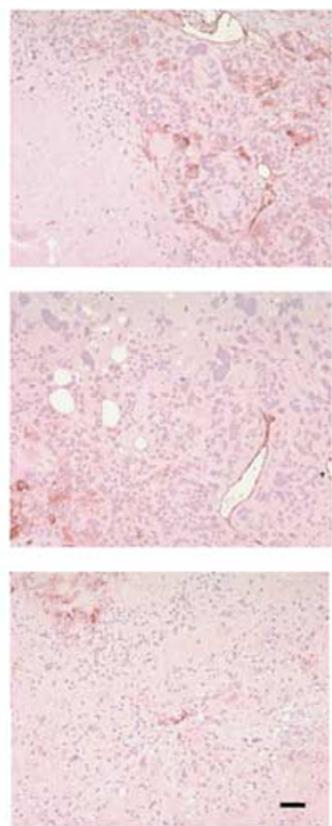

Figure 2 Long-term response monitoring of tumour vasculature. $(\mathbf{A})$ Tumours treated with varying doses of radiation alone $(P<0.00 \mathrm{I}$ for 0 and $8 \mathrm{~Gy}$ showing significant difference in vascularity; P>0.05 for 2 Gy showing insignificant changes in the NVI). (B, C) Low and high ultrasound-activated microbubbles in combination with radiation treatment. $(P<0.00 \mathrm{I}, n=5$, error bars =s.e.m.). (D) Tumour tissue sections were stained with $C D 3 \mathrm{I}$ antibodies, counted and quantified after 3-4 weeks of treatment. Tumours show areas of sparse endothelial cell staining from increasing concentrations of microbubbles and radiation doses. Bar indicates $50 \mu \mathrm{m}$. Magnification $=\times 10$. 

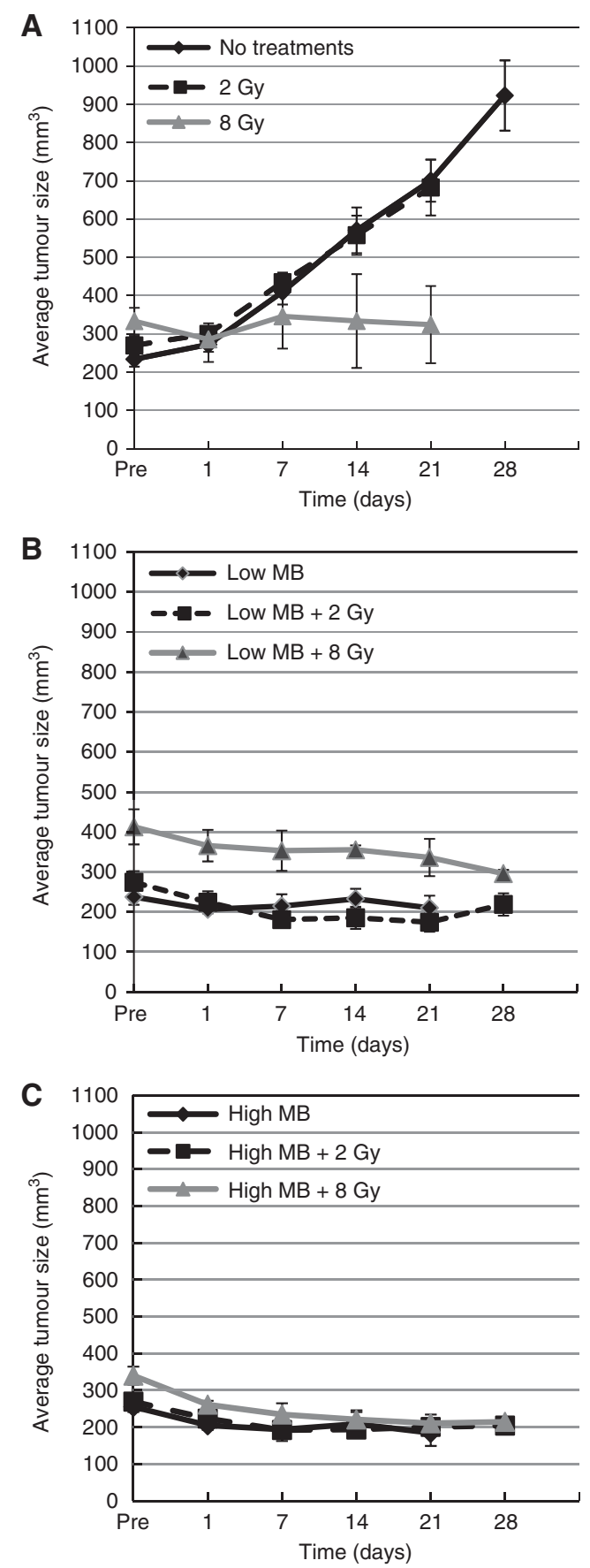

Figure 3 Effects of ultrasound-activated microbubbles and radiation on the growth of HT-I 376 human bladder cancer xenografts in SCID mice. Mice bearing HT- 1376 human bladder xenografts were grouped for various combination treatments with ultrasound-activated microbubbles and radiation. Mice were also divided into control groups consisting of no treatment or non activated microbubbles or with ultrasound only. (A) No significant difference in tumour size for $2 \mathrm{~Gy}$ treatment, however, growth inhibition was observed in $8 \mathrm{~Gy}$ treatment with significant difference $(P<0.001, n=4-5)$. (B, C) Low and high ultrasound-activated microbubbles with varying doses of radiation showed a significant increase in tumour growth inhibition $(P<0.001, n=4-5)$. Error bars $=$ s.e.m.

tumour kill potential. We assessed tumour cell death using immunohistochemistry and measured changes in tumour size at each imaging time point. Treated tumours with combination therapy revealed tumour growth delay (Figure 3). Treated tumours demonstrated significant response to treatment within the tumour's centre region. Circumferential tumour regions showed less significant signs of cell death according to the immunohistochemistry. Hematoxylin and eosin staining was performed on long-term studies and demonstrated an increase in cell death as higher concentrations of ultrasound-driven microbubbles and radiation were combined together (Figure 4B). Short-term treated and untreated tumours revealed insignificant changes in tumour size, however, the effects of treatment were more obvious in the long-term results. Short-term and long-term tumours showed elevated regions of cell death according to immunohistochemical (TUNEL) staining (Figure 4A).

In order to validate treatment effects and confirm power Doppler effects, CD31 immunohistochemical analysis was performed. Power Doppler quantification and immunohistochemistry (CD31) results both demonstrated a decrease in measured vascularity. Cluster of differentiation-31 stains were used to detect presence of endothelial cells in all treated and non-treated groups. Vascular structures were identified and analysed for the presence of viable endothelial cells. Analysis revealed decreased CD31 counts in treated tumours, corresponding with a reduction in the number of viable blood vessels found within the tumour (Figure 5).

\section{DISCUSSION}

In this study, we used high frequency Doppler ultrasound in conjunction with biological methods to monitor tumour response to ultrasound-driven microbubbles and radiation in a bladder cancer xenograft model. We tested the hypothesis that ultrasoundmediated microbubble treatment of tumours can be an effective vascular targeting agent that can also potentiate the effects of radiation therapy in bladder cancer xenografts in vivo. The study demonstrated that ultrasound-stimulated microbubbles in combination with radiation can induce rapid hematopoietic disruption, which presents as tumour cell death within the tumour centre in both short and long-term studies.

Microbubbles sequestered to the interior lumen of tumour vasculature can induce stress effects to the endothelium. When driven by ultrasound, microbubble disruption can result in damage to the endothelium through shear stress. This event can subsequently initiate gross vascular reorganisation within the tumour compartment. Vessel phenotypes found within the tumour compartment are typically porous, leaky, and are architecturally entangled and premature (Jain, 2005). This poses a problem in the delivery of oxygen, a major radiation enhancing molecule. In combination with cytotoxic therapies, vascular targeting agents are showing promise in tumour control by breaking the inter-reliance of cancer cells with endothelial cells in tumours (Thomlinson, 1973; Tiecher et al, 1994). Paris et al (2001) showed that endothelial cells can be preferentially damaged in response to radiation exposure. Kolesnick and Fuks, 2003 supported these findings and identified that ionising radiation activated the sphyngomyelin-ceramide pathway and triggered endothelial cell apoptosis. This same pathway is suspected in playing a pivotal role in radiation response and therefore could influence tumour response. Some previous studies have tested the efficacy of vascular targeting agents and radiation. Blakey et al, 2002 used ZD6126 in combination with radiotherapy and found improved treatment outcome. Kozin et al (2001) used VEGFR2 antibody treatment, an anti-angiogenic agent that interrupts the VEGF pathway in combination with radiation therapy and showed that there was favourable tumour response. In both studies, researchers used molecular targets to inhibit endothelial cell proliferation and found that the result enhanced tumour killing capacity. Our current study implements a biophysical targeting model to abrogate endothelial cells by the way of ultrasound-driven microbubbles. When used in combination with radiation, our results also revealed enhanced tumour killing. 
A

0 Gy

No MB
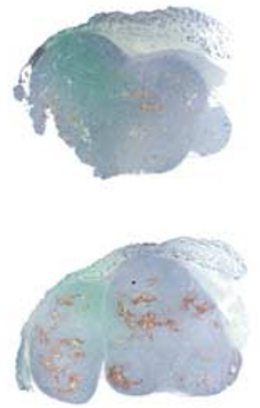

Low MB

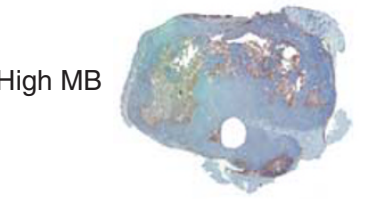

B
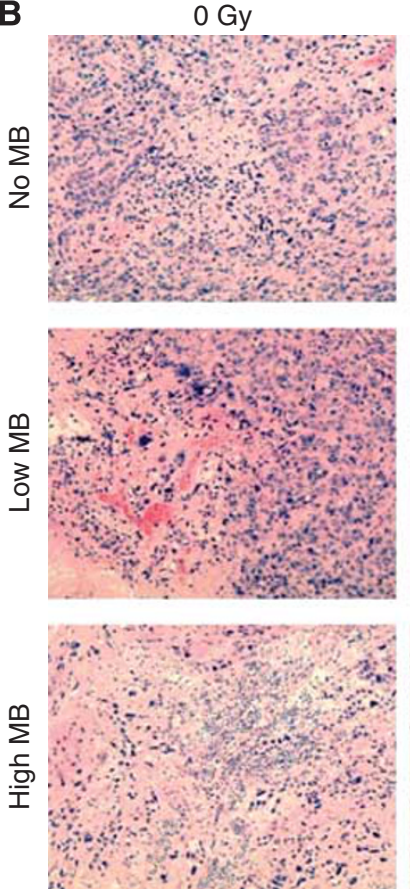

2 Gy
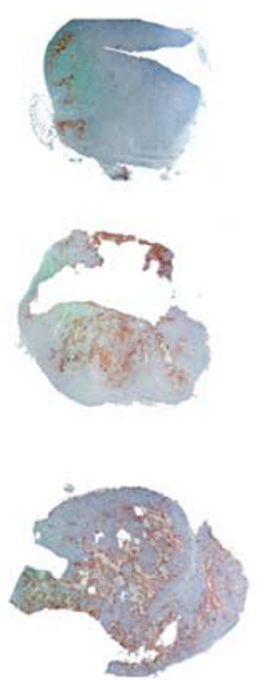

2 Gy
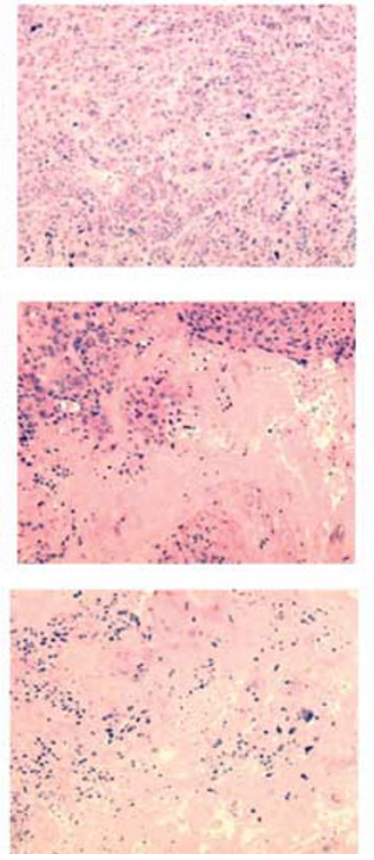

8 Gy
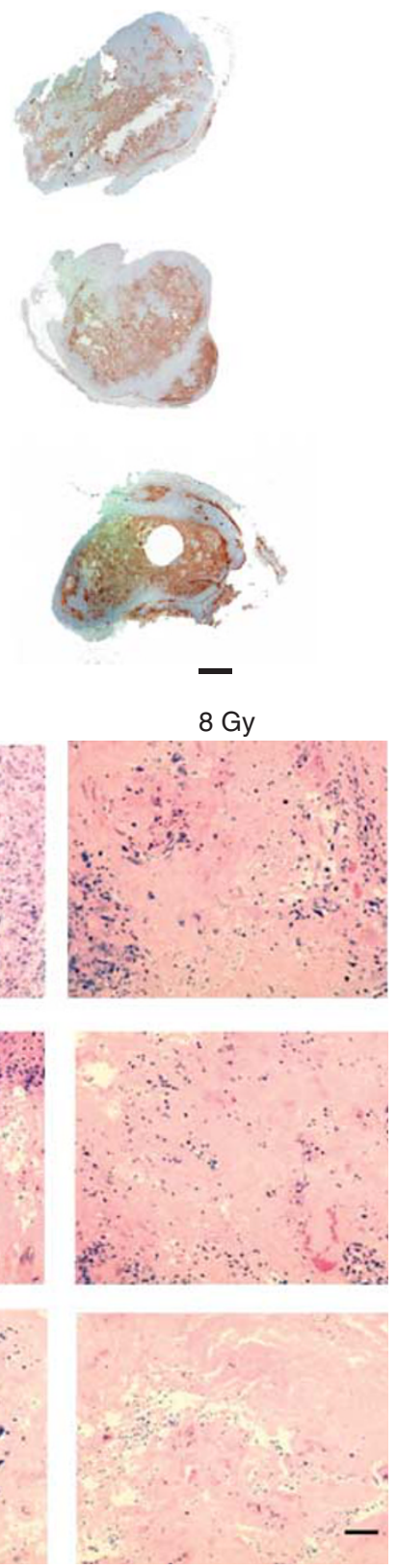

Figure 4 Immunohistochemical analysis in short and long-term tumours. (A) TdT-mediated dUTP-biotin nickend-labelling staining $24 \mathrm{~h}$ after treatment indicates tumour necrosis resulting from local treatment. Radiation treatments alone show condensed necrotic area in multiple foci around the tumour area, which is most apparent after $8 \mathrm{~Gy}$. Radiation treatments in combination with low microbubble administration exhibiting viable cells at tumour periphery. High-concentration microbubble treatments with varying doses of radiation. Severe necrotic areas observed at tumour periphery and development of cystic spaces. Scale Bar indicates $2 \mathrm{~mm}$. (B) Hematoxylin and eosin staining 3-4 weeks after treatment $\times 20$ magnification at core regions of tumours. Aberrations to the tumour microenvironment as a result of local tumour ischaemia. Top row. H\&E staining through the tumour specimens demonstrated expected results with single-fraction radiation treatment of $2 \mathrm{~Gy}$ showed no obvious regions of cell death. Tumour cells were homogenous and evenly dispersed. With $8 \mathrm{~Gy}$, cells were sparse and showed regions of decreased density, suggesting cell death. Middle row, post irradiation micropetechiae as a result of microbubble treatment response. Ultrasound-activated microbubble treatment caused a heterogeneous appearance consisting of fewer intact cells at the centre and circumscribed by healthy cells at the periphery. Bottom row, post irradiation with $3 \%$ microbubbles resulting in tumour cell necrosis at the centre. Tumours treated with combined microbubble-ultrasound and radiation demonstrated the largest areas of cell death. Bar indicates $50 \mu$ m; magnification $(\times 20)$.

Other studies using imaging have also utilised blood flow imaging for such studies. Goertz et al (2002) showed a reduction in doppler signals, and thus reduced blood flow perfusion within the tumour after administering an anti-vascular agent ZD126 as a monotherapy to human melanoma xenograft bearing mice. Their group demonstrated an optimal reduction in vasculature at $\sim 4 \mathrm{~h}$ after treatment administration and effectively presented the usefulness of Doppler sonography in monitoring vascular response. The variability in the study here has been minimised through careful handling of animals for experiments and ensuring consistent animal ages, weights and tumour sizes. Animals were treated and imaged as groups to also minimise variability. Consistency in anaesthesia and animal temperature also likely contributed to the low variability in measured Doppler signals present in this study. 
A

No MB
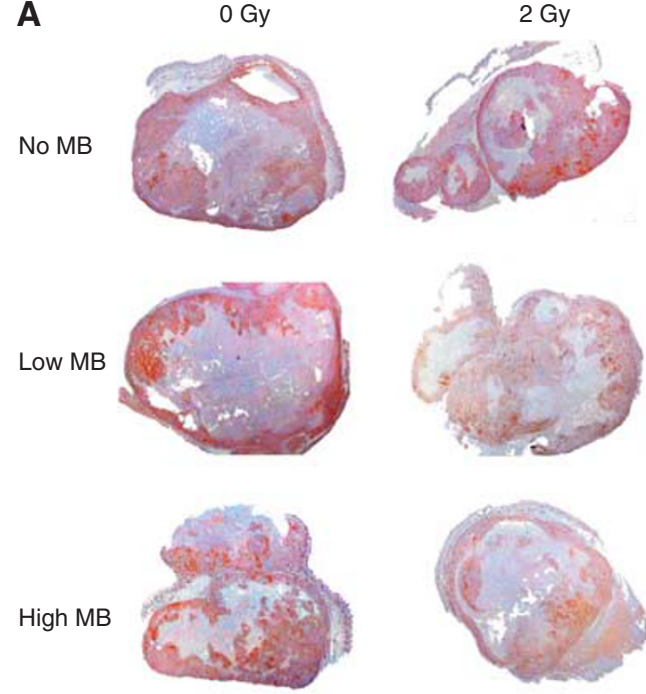

8 Gy
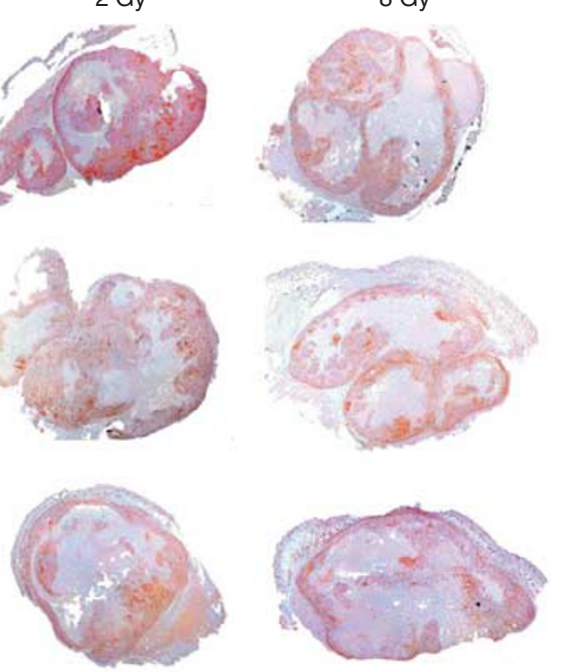
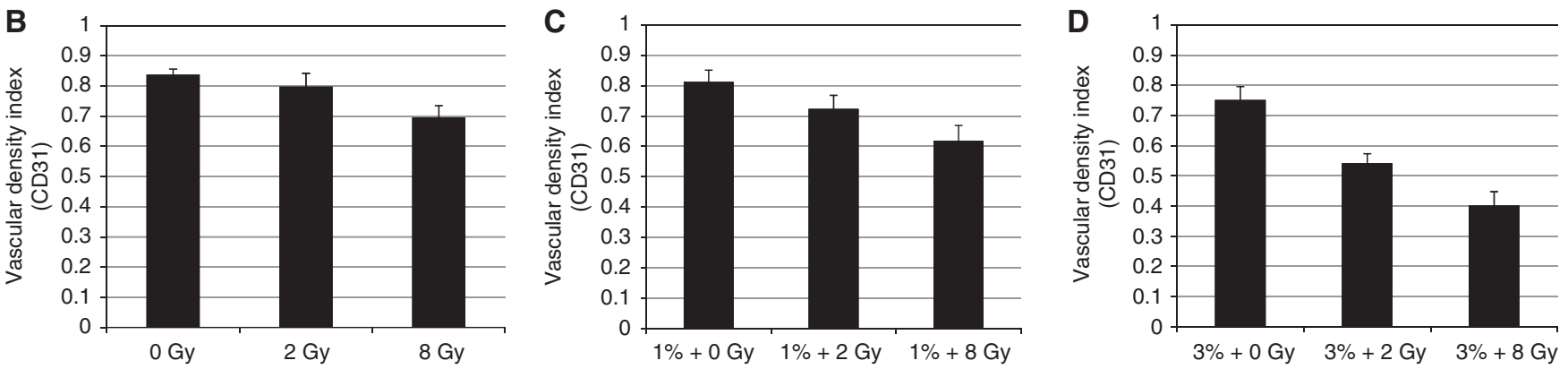

Figure 5 Long-term CD3I staining for endothelial cell markers in tumours. Cluster of differentiation-3I antibody staining was counted and quantified in tumours with varying treatments of ultrasound-activated microbubbles and radiation. (A) Cluster of differentiation-3I staining was performed for tumours $24 \mathrm{~h}$ after treatment. A reduction in the total staining of endothelial cells was observed in tumours. Vascular remnants were seen with the absence of endothelial cells. Bar indicates $2 \mathrm{~mm}$. (B) Mice bearing human bladder HT- 1376 xenografts treated with varying doses of radiation show reduced endothelial populations $21-28$ days after treatment $(P>0.05, n=5)$. (C) Low-concentration ultrasound-activated microbubbles in combination with radiation show reduction in vasculature $(P>0.05$ (microbubbles alone), $(P<0.01$ low microbubbles + radiation), $n=5)$. (D) High-concentration ultrasound-activated microbubbles in combination with varying radiation doses show decrease in endothelial cells at $2 \mathrm{~Gy}(P>0.05, n=5)$ and significant difference at $8 \mathrm{~Gy}$ treatments $(P<0.01, n=5)$ after $21-28$ days of treatment. Error bars $=$ s.e.m.

Radiation treatments present several limitations in achieving optimal therapeutic outcomes because of tumour cell heterogeneity. As a result, many regimens require varying doses of radiation in order to control tumour growth and also to make a significant effect on the tumour vasculature. Our results indicated such a complication in the delivery of only $2 \mathrm{~Gy}$ of radiation to the tumour, demonstrating that tumour control at low doses may have weak effects on controlling the tumour, but that it can also induce pro-vascular effects through angiogenic signalling. We suspect that this induction of vascularisation through low dose radiation alone is caused by the MAP-K dependant pathway described by Park et al, 2001. Interestingly, when $2 \mathrm{~Gy}$ radiation was combined with low and high concentrations of ultrasound-driven microbubbles, vessel disruption was observed in tumours. Vascular disrupting agents serve as a feasible model in combination with cytotoxic agents such as radiation because targeting vasculature is independent of tumour type and can overcome the obstacles that cytotoxic drugs have in combating cell heterogeneity. Furthermore, tumour cells are more likely to resist cytotoxic therapies because of their genetic instability (Feron, 2004), and thus targeting the endothelial cells can be beneficial because of its relative accessibility to vascular targeting agents, its low potential to disrupt normal tissue and its ability to influence death on tumour clonogens that are dependent on vessels (Wachsberger et $a l, 2003)$. Ultrasound-driven microbubbles used as vascular disrupting agents can have many advantages to molecular-based targeting. For instance, VEGF-antibody based agents could potentially have unwanted effects on other biological pathways as VEGF expression is also found in certain types of hematopoietic and stromal cells (Duda et al, 2007). Therefore, the added advantage to using ultrasound-driven microbubbles to perturb endothelial cells is its disposition to be locally targeted, thereby minimising the influence on other biochemical pathways found in distant physiological processes.

Our ultrasound imaging assessment and immunohistological analysis demarcated a reduction in the vasculature and demonstrated progressive regions of apoptotic cell death within the centre of the tumour. In comparison with other anti-vascular approaches that have been shown to exhibit vascular regeneration after 7 days (Red-Horse and Ferrara, 2006), we observed no such recovery in our tumours when ultrasound-stimulated microbubbles were used in combination with radiation. Our ultrasound scans and histological analysis revealed differential vascular response around the tumour's periphery. This circumferential vascular pattern demonstrated stable vascular abundance in contrast to the tumour's core regions that revealed transient and sparse vasculature. We propose that this phenomenon is attributed to the vascular growth pattern before treatment. Xuan et al, 2007 used power Doppler ultrasound to detect angiogenic blood flow in prostate tumours and quantified vessel diameters of $100 \mu \mathrm{m}$ or larger in the inner core and $12-24 \mu \mathrm{m}$ vessels around the tumour periphery. Larger vessels feeding the centre of the tumour would 
result in better centre perfusion with microbubbles and may explain why vascular targeting agents tend to be well sequestered in the tumour's core. The centre region not only showed areas of vascular atrophy but was also circumscribed by tumour necrosis. These findings may be a possible result of tumour cell starvation and a result of direct and indirect radiation targeting.

\section{CONCLUSION}

Vascular targeting agents can be an effective partnering modality in eradicating the tumour stroma. The current study suggests that ultrasound-mediated microbubbles can be successfully used as a vascular targeting agent and can enhance radiosensitivity in bladder cancer carcinoma. Endothelial cell disruptions and direct tumour cell kill are suspected to be implicated in both processes that render the tumour necrotic. Furthermore, this study emphasises the importance of considering multi-modality approaches in optimising the therapeutic ratio and draws attention to the importance of vascularity and endothelial cell interactions with radiosensitivity.

\section{ACKNOWLEDGEMENTS}

This research project was funded by the American Association of Cancer Research (Henry Shepard Bladder Cancer Research Grant) and the Terry Fox Foundation. We thank Michelle Martin, RVT from Comparative Research at Sunnybrook Health Sciences Centre for her expertise in animal care and exceptional technical support.

\section{REFERENCES}

Blakey DC, Westwood R, Walker M, Hughes GD, Davis PD, Ashton SE, Ryan AJ (2002) Antitumour activity of the novel vascular targeting agent ZD6126 in a panel of tumour models. Clin Cancer Res 8: 1974-1983

Cooney MM, van Heekcheren W, Bhakta S, Ortz J, Remick SC (2006) Drug insight: vascular disrupting agents and angiogenesis-novel approaches for drug delivery. Nature Clin Prac Oncol 12: 682-692

Dings RP, Loren M, Heun H, McNiel E, Griffioen AW, Mayo KH, Griffin RJ (2007) Scheduling of radiation with angiogenesis inhibitors anginex and avastin improves therapeutic outcome via vessel normalization. Clin Cancer Res 13(11): 3395-3402

Duda DG, Batchelor TT, Willett CG, Jain RK (2007) VEGF-targeted cancer therapy strategies: current progress, hurdles and future prospects. Trends Mol Med 13(6): 223-230

Feron O (2004) Targeting the tumour vascular compartment to improve conventional cancer therapy. Trends Pharmacol Sci 25(10): 536-542

Fukumura D, Jain RK (2007) Tumour microenvironment abnormalities: causes, consequences, and strategies to normalize. J Cell Biochem 101: 937-949

Gadgeel SM, LoRusso PM, Wozniak AJ, Wheeler C (2002) A dose-escalation study of the novel vascular-targeting agent ZD6126, in patients with solid tumors. Proc Am Soc Clin Oncol 21: 438

Goertz DE, Yu JL, Kerbel RS (2002) High Frequency Doppler Ultrasound Monitors the Effects of Antivascular Therapy on Tumor Blood Flow. Cancer Res 62: 6371-6375

Hamper UM, DeJong MR, Caskey CI, Sheth S (1997) Power Doppler imaging: clinical experience and correlation with color Doppler US and other imaging modalities. RadioGraphics 17: 499-513

Hayes AJ, Li LY, Lippman ME (1999) Antivascular therapy: a new approach to cancer treatment. $\mathrm{Br}$ Med J 318: 853-8553

Jain RK (2005) Normalization of tumour vasculature: an emerging concept in antiangiogenic therapy. Science 307: 58-62

Jameson MB, Thompson PI, Baguely BC, Evans BD, Harvey VJ, Porter DJ, McCrystal MR, Small M, Bellenger K, Gumbrell L, Halbert GW, Kestell P (2003) Clinical aspects of a phase I trial of 5, 6-dimethylxanthenone-4acetic acid (DMXAA), a novel antivascular agent. Br J Cancer 88: 1844-1850

Karshafian R, Bevan PD, Williams R, Samac S, Burns PN (2009) Sonoporation by ultrasound activated microbubble contrast agents: effect of acoustic exposure parameters on cell membrane permeability and cell viability. Ultrasound Med Biol 35(5): 847-860

Klibanov AL (2006) Microbubble contrast agents: Targeted ultrasound imaging and ultrasound-assisted drug delivery applications. Invest Radiol 41(3): 354-362

Kobayashi N, Yasu T, Yamada S, Kudo N, Kuroki M, Kawakami M, Miyatake K, Saito M (2002) Endothelial cell injury in venule and capillary induced by contrast ultrasonography. Ultrasound Med Biol 28(7): 949-956

Kolesnick R, Fuks Z (2003) Radiation and ceramide-induced apoptosis. Oncogene 22: 5897-5906

Kozin SV, Boucher Y, Hicklin DJ, Bohlen P, Jain RK, Suit HD (2001) Vascular endothelial growth factor receptor-2-blocking antibody potentiates radiation-induced long-term control of human tumour xenografts. Cancer Res 61: 39-44

Nieder C, Wiedenmann N, Andratschke N, Molls M (2006) Current status of angiogenesis inhibitors combined with radiation therapy. Cancer Tretment Rev 32: 348-364

Nishida N, Yano H, Nishida T, Kamura T, Kojiro M (2006) Angiogenesis in cancer. Vasc Health Risk Manage 2(3): 213-219

O'Reilly MS (2006) Radiation combined with Antiangiogenic and Antivascular Agents. Semin Radiat Oncol 16: 45-50

Park JS, Qiao L, Su ZZ, Hinman D, Willoughby K, McKinstry R, Yacoub A, Duigou GJ, Young CS, Grant S, Hagan MP, Ellis E, Fisher PB, Dent P (2001) Ionizing radiation modulates vascular endothelial growth factor (VEGF) expression through multiple mitogen activated protein kinase dependent pathways. Oncogene 20: 3266-3280

Paris F, Fuks Z, Kang A, Capodieci P, Juan G, Ehleiter D, HaimovitzFriedman A, Cordon-Cardo C, Kolesnick R (2001) Endothelial apoptosis as the primary lesion initiating intestinal radiation damage in mice. Science 293: 293-297

Raben D, Ryan A (2005) Vascular-targeting agents and radiation therapy in lung cancer: Where do we stand in 2005? Clin Lung Cancer 7(3): 175-179

Radema SA, Beerepoot LV, Witteveen PO, Gebbink MF, Wheeler C, Voest EE (2002) Clinical evaluation of the novel vascular-targeting agent ZD6126: assessment of toxicity and surrogate markers of vascular damage. Proc Am Soc Clin Oncol 21: 439

Red-Horse K, Ferrara N (2006) Imaging tumour angiogenesis. J Clin Invest 116: $2585-2587$

Rustin GJ, Bradley C, Galbraith S, Stratford M, Loadman P, Waller S, Bellenger K, Gumbrell L, Folkes L, Halbert G (2003) 5,6-dimethylxanthenone-4-acetic-acid (DMXAA), a novel antivascular agent: phase I clinical and pharmacokinetic study. Br J Cancer 88: 1160-1167

Thomlinson RH (1973) Radiation and the vascularity of tumours. Br Med Bull 29(1): 29-32

Tiecher BA, Dupuis N, Kusomoto T, Robinson MF, Liu F, Menon K, Coleman CN (1994) Antiangiogenic agents can increase tumour oxygenation response to radiation therapy. Radiat Oncol Invest 2(6): 269-276

Thorpe PE (2004) Vascular targeting agents as cancer therapeutics. Clin Cancer Res 10: 415-427

Wachsberger P, Burd R, Dicker AP (2003) Tumour response to ionising radiation combined with antiangiogenesis or vascular targeting agents: Exploring mechanisms of interaction. Clin Cancer Res 9: 1957-1971

Xie F, Tsutsi JM, Lof J, Unger EC, Johanning J, Culp WC, Matsunaga T, Porter TR (2005) Effectiveness of lipid microbubbles and ultrasound in declotting thrombosis. Ultrasound Med Biol 31(7): 979-985

Xuan JW, Bygrave M, Jiang H, Valiyeva F, Dunmore-Buyze J, Holdsworth DW, Izawa JI, Bauman G, Moussa M, Winter SF, Greegnberg NM, Chin JL, Drangova M, Fenster A, Lacefield JC (2007) Functional neoangiogenesis imaging of genetically engineered mouse prostate cancer using three dimensional power doppler ultrasound. Cancer Res 67(6): 2830-2839

Yu T, Wang G, Hu K, Ma P, Bai J, Wang Z (2004) A microbubble agent improves the therapeutic efficiency of high intensity focused ultrasound: a rabit kidney study. Urol Res 32: 14-19

This work is published under the standard license to publish agreement. After 12 months the work will become freely available and the license terms will switch to a Creative Commons Attribution-NonCommercial-Share Alike 3.0 Unported License. 